\title{
Impact of Scaling Range on the Effectiveness of Detrending Methods
}

\author{
D. GRECH* AND Z. MAZuR \\ Faculty of Physics and Astronomy, University of Wrocław, Econophysics and Time Series Analysis Group (ETSA), \\ pl. M. Borna 9, PL-50204 Wrocław, Poland

\begin{abstract}
We make the comparative study of scaling range properties for detrended fluctuation analysis (DFA), detrended moving average analysis (DMA) and recently proposed new technique called modified detrended moving average analysis (MDMA). Basic properties of scaling ranges for these techniques are reviewed. The efficiency and exactness of all three methods towards proper determination of scaling Hurst exponent $H$ is discussed, particularly for short series of uncorrelated and persistent data.
\end{abstract}

DOI: 10.12693/APhysPolA.127.A-59

PACS: 05.45.Tp, 89.75.Da, 02.60.-x, 89.20.-a

\section{Introduction}

In last years much effort was put into precise analysis of scaling range for power laws used to classify long-term memory properties in complex systems and in time series [1-7]. This effect in stationary time series $x_{t}$, $(t=1, \ldots, L)$, is usually attacked with two point autocorrelation function $C_{s}=\left\langle\Delta x_{t} \Delta x_{t+s}\right\rangle$, where \langle\rangle is the average taken from all data in a signal with increments $\Delta x_{t}=x_{t+1}-x_{t}$. The following scaling law is proven to be valid for the main model of stationary data with long term memory, i.e., the fractional Brownian motion $(\mathrm{fBm})[8,9]$

$$
C_{s} \simeq \frac{1}{2}(2-\gamma)(1-\gamma) s^{-\gamma},
$$

where $s$ is the time lag and the autocorrelation scaling exponent $\gamma(0 \leq \gamma \leq 1)$ describes the level of long term memory in a signal. The two edge values $\gamma=0$ and $\gamma=1$ are related respectively to fully correlated or uncorrelated (integer Brownian motion) data, leaving space for other fractional persistent Brownian motion $(0<\gamma<1)$ in between.

A direct calculation of correlation functions and $\gamma$ exponent may suffer for real data from problems connected with noise present in time series or possible nonstationarities in data (locally changing the quality of power law in Eq. (1)). Therefore, it is recommended to reduce these effects not calculating $\gamma$ directly, but studying the "integrated profile" of the data, i.e., the random walk $x_{t}$ instead of $\Delta x_{t}$ behavior. In the latter case the scaling Hurst exponent $H$ of the series $[10,11]$ is measured. Traditionally, $H$ is defined as the exponent of the power law relation

$$
\operatorname{var}\left(x_{t}\right)=\left\langle x_{t}^{2}\right\rangle-\left\langle x_{t}\right\rangle^{2} \simeq t^{2 H}
$$

where $\operatorname{var}()$ is the variance calculated for a process of length $t$. All methods calculating $H$ are useful in analysis

\footnotetext{
*corresponding author; e-mail: dgrech@ift.uni.wroc.pl
}

of long term memory properties in data since there exists a simple formula proven for $\mathrm{fBm}$ which links $\gamma$ and $H$ exponent for large $t[12]$ :

$$
H=1-\frac{\gamma}{2} \text {. }
$$

Furthermore, to avoid an artificial bias in $x_{t}$ data caused by the presence of trend influencing the final outcome for $H$, the so called detrending procedure is recommended. Two efficient major techniques were proposed in literature to do so: detrended fluctuation analysis (DFA) $[13,14]$ and detrended moving average analysis (DMA) [15-18] with variety of their 'clones' applicable also for multifractals [19], where two point autocorrelation functions are not sufficient to describe the variety of autocorrelation properties in data. Recently DMA was generalized also to its modified version called MDMA [20] where the statistics of data points used to calculate the trend in signal is more balanced than in a case of DMA.

The scaling law from Eq. (1) has been built into DFA in a form of power law

$$
F^{2}(\tau) \simeq \tau^{2 H},
$$

where $F^{2}(\tau)$ is the averaged squared fluctuation of the signal around its local trend in time windows of fixed length $\tau$. To be more precise:

$$
F^{2}(\tau)=\frac{1}{N} \sum_{k=1}^{N} \hat{F}^{2}(\tau, k),
$$

where

$$
\hat{F}^{2}(\tau, k)=\frac{1}{\tau} \sum_{j=1}^{\tau}\left\{x_{(k-1) \tau+j}-P_{j, k}\right\}^{2} .
$$

Here $N=[L / \tau]^{\dagger}$ stands for the number of nonoverlapping boxes obtained after cutting the whole walk $x_{t}(t=1, \ldots L)$ into separate pieces where detrending is performed with a polynomial trend $P_{j, k}$ fitted to data in $k$-th window box. The sum in Eq. (5) runs from the

\footnotetext{
${ }^{\dagger}[$ means the integer part.
} 
oldest to newest data in series ${ }^{\ddagger}$. If $p$-th order polynomial is taken for detrending in DFA, the notation DFA- $p$ is often used.

The DMA method serves the similar power law

$$
F_{D M A}^{2}(\lambda) \sim \lambda^{2 H}
$$

but here the fluctuation function (variance of detrended signal) is defined according to

$$
F_{D M A}^{2}(\lambda)=\frac{1}{L-\lambda+1} \sum_{t=\lambda}^{L}\left(x_{t}-\left\langle x_{t}\right\rangle_{\lambda}\right)^{2},
$$

where $\left\langle x_{t}\right\rangle_{\lambda}$ plays the role of a trend. The latter one is defined as the moving average of length $\lambda$ calculated as

$$
\left\langle x_{t}\right\rangle_{\lambda}=\frac{1}{\lambda} \sum_{k=t-\lambda+1}^{t} x_{k} .
$$

The DMA method suffers however from diversified statistics of data points contributing to fluctuation function $F_{D M A}^{2}(\lambda)$, since only data points $x_{t}$ with $t \geq \lambda$ can be taken into account for determination of the variance $F_{D M A}^{2}(\lambda)$. Thus the statistics depends here strongly on the chosen length $\lambda$ of the moving average. For the particular choice of $\lambda$ and $L$ only $L-\lambda+1$ detrended values contributes to the $F_{D M A}^{2}(\lambda)$ in the power law determined by Eq. (7).

This difficulty can be omitted in the modified DMA technique (MDMA) [20]. Such a modification is based on the assumption that usually more than $L$ data points actually exist in a real time series one investigates and some amount of data stored before the basic series of length $L$ - although not explored - is basically also available for a study. The available full amount of data can therefore be written as the sequence $\left\{x_{-\lambda_{\max }}, \ldots, x_{-2}, x_{-1}, x_{1}, x_{2}, \ldots, x_{L}\right\}$, where $\lambda_{\max }$ is the maximal scaling range used in particular calculation of $H$. Thus one is able to calculate trends (moving averages) also for those data points where DMA procedure with particular choice of $\lambda$ simply fails. To be precise, Eq. (8) is replaced in MDMA by

$$
F_{M D M A}^{2}(\lambda)=\frac{1}{L} \sum_{t=1}^{L}\left(x_{t}-\widetilde{\left\langle x_{t}\right\rangle_{\lambda}}\right)^{2},
$$

with the moving average of length $\lambda$ calculated for $t \geq \lambda$ according to Eq. (9), while modified for $0<t<\lambda$ as

$$
\widetilde{\left\langle x_{t}\right\rangle_{\lambda}}=\frac{1}{\lambda}\left(\sum_{k=1}^{t} x_{k}+\sum_{k=t-\lambda}^{-1} x_{k}\right),
$$

where $k<0$ means that summation takes into account also additional data points preceding the basic series. The power law similar to Eq. (7) is still expected in MDMA where $F_{D M A}^{2}(\lambda)$ is replaced by $F_{M D M A}^{2}(\lambda)$, i.e.

\footnotetext{
$\ddagger$ Summation in both directions — along the time axis and back — is also used to avoid problems with the edge data not entering any of $N$ time boxes; an average of $F^{2}(\tau)$ calculated in these two directions is used then.
}

$$
F_{M D M A}^{2}(\lambda) \sim \lambda^{2 H} .
$$

An exemplary plot of dependence between detrended fluctuation function and the size of time box $\tau$ (or the length of moving average $\lambda$ ) is presented in log-log plots for three discussed methods in Figs. 1, 2 for the simplest case of two different lengths of synthetic uncorrelated data $(H=1 / 2)$. Already here differences in scaling features between three methods are easy observed. MDMA seems to be more robust to deviation from scaling for larger $\lambda$ than DMA. Simultaneously, DFA $\S$ exhibits qualitatively worse scaling property than two other methods - particularly for short time series. This behavior should however be examined more systematically and on the base of strict quantitative investigations.



Fig. 1. Comparison of detrended fluctuations in DFA, DMA and MDMA versus size of window box $(\tau)$ or the length of moving average $(\lambda)$ for uncorrelated synthetic data mimicking integer Brownian motion of length $L=$ 1000 steps. The plots are artificially shifted relative to each other to make differences between them more visible.



Fig. 2. Same as in Fig. 1 but for the integer Brownian motion of length $L=6000$ steps. wise.

$\S$ DFA-1 has been used within this article unless stated other- 


\section{Methodology and Data Analysis}

The precise value of the scaling range for power laws given by Eqs. (4), (7) and (12) will be a function of available length of a signal $L$, i.e., $\lambda=\lambda(L)$ and $\tau=\tau(L)$ but obviously they will depend also on the accuracy of fit $R^{2}$ of the scaling law - usually represented in log$\log$ scale as a linear regression fit. One expects also that dependence on the persistency level in data may occur. Hence, the general analysis of this problem may be quite complex and should be made step by step with convincing statistics of synthetically generated time series with a priori known autocorrelation properties. The persistent time series of $\mathrm{fBm}$ (as the basic model of real data with long term memory) can be generated using the Fourier filtering (FFM) algorithm [21]. The scaling behavior of two point autocovariance function $C_{s}=\left\langle\Delta x_{t} \Delta x_{t+s}\right\rangle$ can then be checked qualitatively and quantitatively as in Ref. [22].
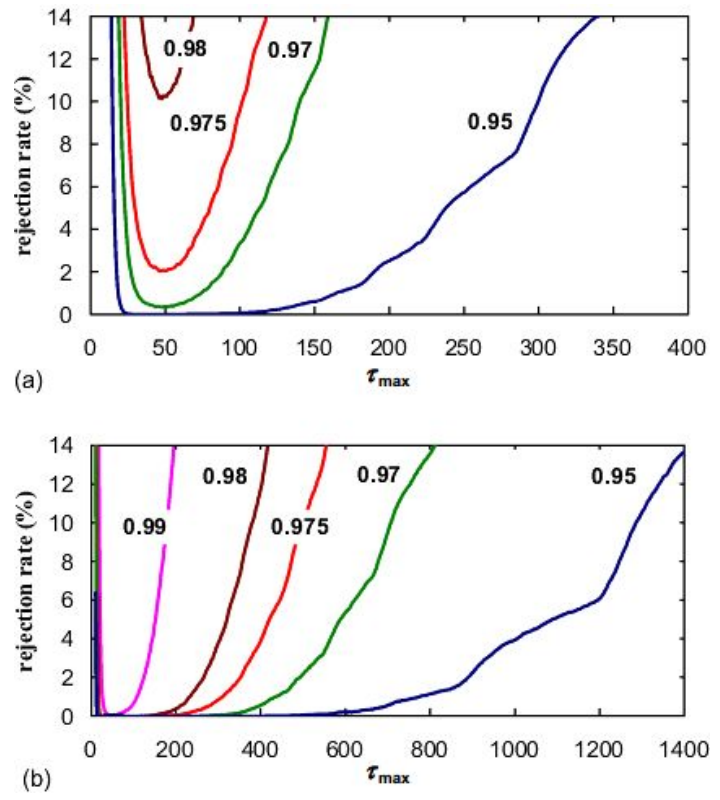

Fig. 3. "Iso- $R$ " curves for DFA-1 - an example of uncorrelated data of length $L=1000$ (top) and $L=3000$ (bottom). For detailed explanations see the main text.

The starting point are plots like in Figs. 3-4. We may call them "Iso-R" curves, since they present the rejection rate in an ensemble of synthetic time series which are below the specified level of Pearson correlation coefficient $R^{2}$ for linear regression plots made in log-log scale according to Eqs. (4), (7) and (12). This rejection rate obviously depends on the maximal scaling range one takes into account. Hence, the larger scaling range is assumed the bigger rejection rate occurs at given $R^{2}$. One may obtain numerous relations from these figures to produce dependencies shown in the remaining plots in Figs. 5-8 from which the principal answers can be deduced. Not all plots for variety of possible parameters are presented here because they look qualitatively similar.

The detailed study of dependencies shown in log-log scale in Figs. 5-6 convince that the final relationship
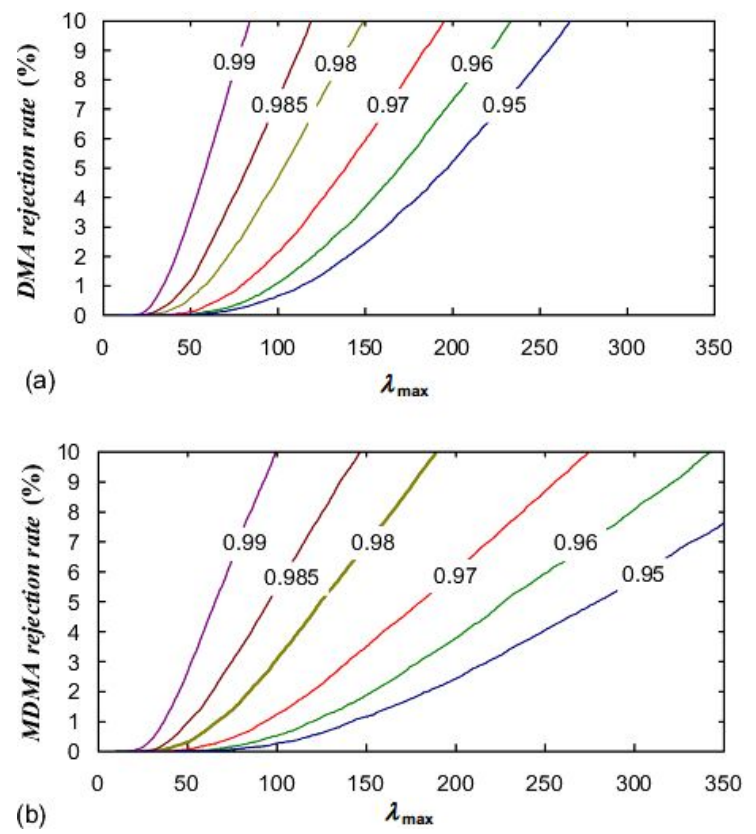

Fig. 4. Similar example as in Fig. 3 but for DMA (top) and MDMA (bottom) applied to integer Brownian motion of length $L=3000$ steps.
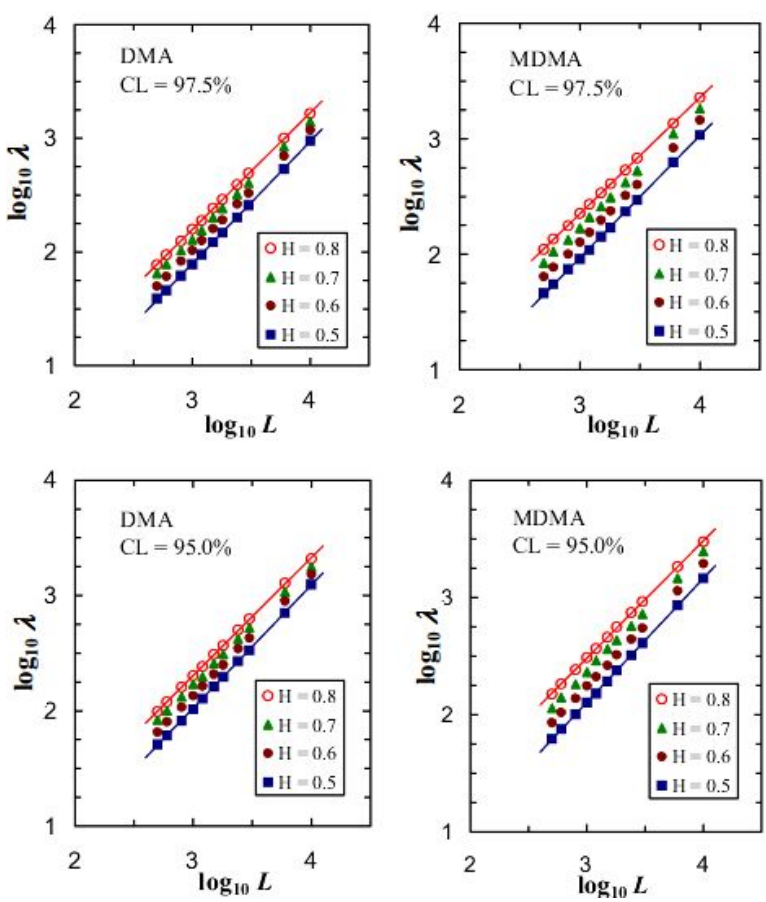

Fig. 5. Dependence between scaling range $\lambda$ and length of time series $L$ for various levels of autocorrelation in data (measured by $H$ exponent). The plots are drawn for particular choice $R^{2}=0.98$ for two confidence levels $C L=97.5 \%$ and $C L=95 \%$. For other $R^{2}$ values (not shown) they look qualitatively similar. The fitting lines are drawn only for edge values $H=0.5$ and $H=0.8$ to make all remaining dependencies more readable. 

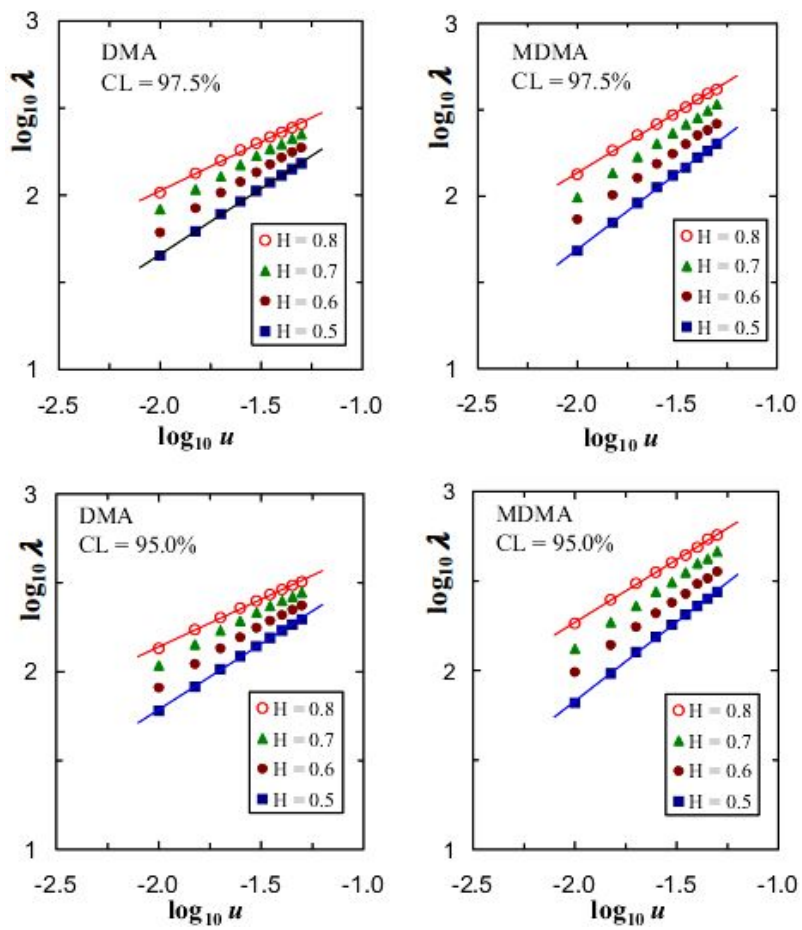

Fig. 6. Same as in Fig. 5 but for a dependence of scaling range on $R^{2}\left(u=1-R^{2}\right)$ for signals of length $L=10^{3}$.

between scaling range $\lambda$, signal length $L$ and the accuracy of fit $R^{2}$ takes both for DMA and MDMA in fBm the power law form

$$
\left.\lambda\left(L, R^{2}\right)\right)=D L^{\eta}\left(1-R^{2}\right)^{\xi},
$$

where $D, \eta$ and $\xi$ depend only on the method (DFA, MDMA) and on the persistency level in data (see, Ref. [20] for details). Moreover, the fitted values of these parameters are linear in $\gamma$ exponent (as well as in $H$ ) in the first approximation. These values are accommodated in Table I and Table II for two confidence levels $(C L=97.5 \%$ and $C L=95 \%)$.

A similar consideration for DFA leads to plots like in Figs. 7-8. On the contrary, they are prepared this time in linear scale and support the relationship

$$
\lambda\left(L, R^{2}\right)=\left(A R^{2}+A_{0}\right) L+B,
$$

where parameters $A, A_{0}$ and $B$ also depend only on the persistency of data and are linear with $\gamma$ for a wide range of $0<\gamma<1$ [23]. The results of corresponding fit for these parameters are given in Table III. The above formulas obtained for $\mathrm{fBm}$ serve the maximal scaling range one may expect in real time series while the local nonstationarities and additive noise may make this range shorter.

However, the knowledge of $\lambda\left(L, R^{2}\right)$ dependence is still not sufficient for practical use, since we do not know whether the scaling exponent $H$ is properly reproduced, even if the scaling law of Eqs. (4), (7) or (12) is firmly confirmed for given scaling range $\lambda$. Therefore, it is worth discussing the efficiency of all three methods in precise determination of Hurst exponents when a precisely determined scaling range is taken for calculations.
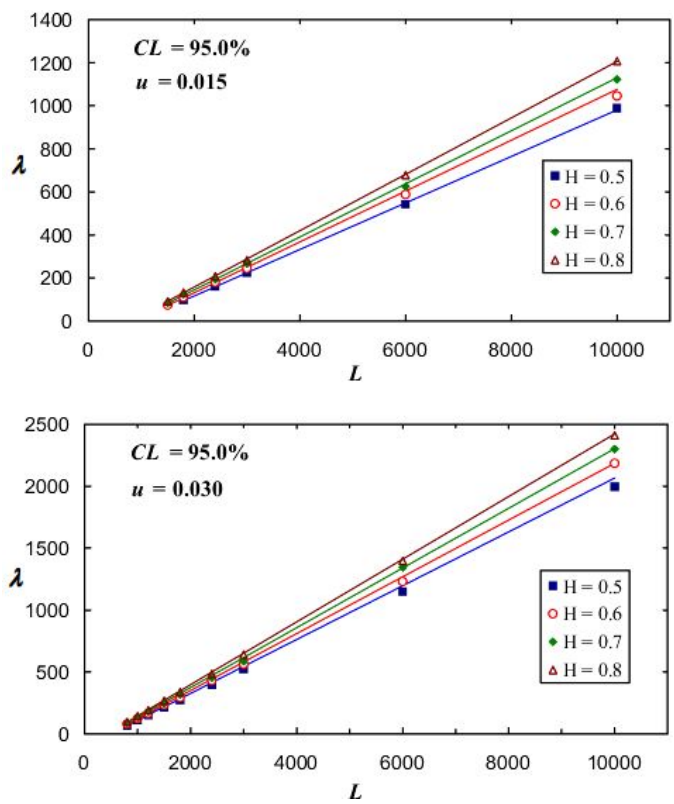

Fig. 7. Dependence of scaling range $\lambda$ on data length for DFA-1 for two particular values of $R^{2}$ at the confidence level $95 \%$. The plots look qualitatively the same for wide range of other $u=1-R^{2}$ (not shown). Perfect linear dependence is observed.
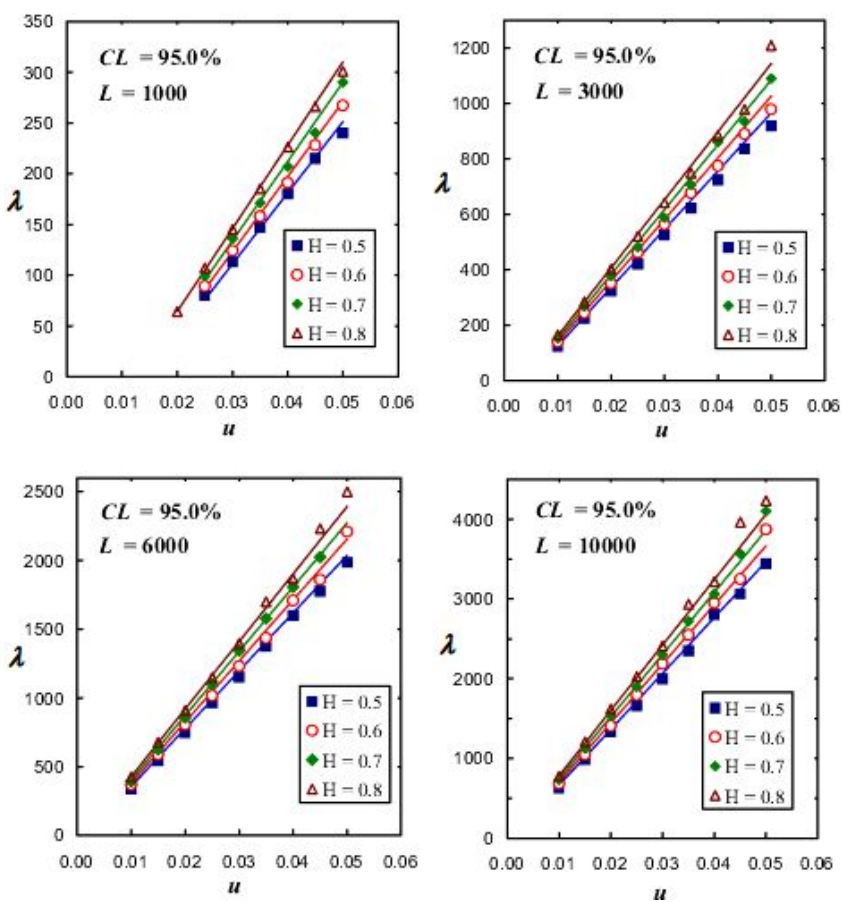

Fig. 8. Same as in Fig. 7 but for dependence on $u=$ $1-R^{2}$ for various signal lengths.

Many particular approaches can be proposed for a such project. Here we provide results obtained for the following questions: (a) how will the outcome (measured value of $H$ exponent in DFA, DMA or MDMA) depend on the chosen scaling range $\lambda$ for synthetic fBm series of data with precisely given autocovariance exponent $\gamma$ 
(or $H$ ) as the input? (b) what is the scaling range of all three methods, most effectively reproducing this input value $H_{\text {in }}$ at the assumed confidence level (we assumed in this approach $\left.\left|H-H_{\text {in }}\right| / H_{\text {in }} \leq 1 \%\right)$ ?

\section{TABLE I}

Results of the best fit for coefficients in Eq. (13) describing scaling range for DMA. The fit is done for series with various autocorrelation level measured by $H$ exponent and for chosen two confidence levels $C L: 97.5 \%$ and $95 \%$. The mean average error $\left(\Delta_{M A E}\right)$ and the maximal error $\left(\Delta_{M E}\right)$ of a fit is also given.

\begin{tabular}{c|c|c|c|c|c|c|c|c|c|c}
\hline \hline$H \backslash C L$ & $D^{97.5 \%}$ & $\eta^{97.5 \%}$ & $\xi^{97.5 \%}$ & $\Delta_{M A E}^{97.5 \%}$ & $\Delta_{M E}^{97.5 \%}$ & $D^{95 \%}$ & $\eta^{95 \%}$ & $\xi^{95 \%}$ & $\Delta_{M A E}^{95 \%}$ & $\Delta_{M E}^{95 \%}$ \\
\hline$H=0.5$ & 0.879 & 1.062 & 0.723 & $1.4 \%$ & $4.2 \%$ & 0.961 & 1.064 & 0.680 & $1.6 \%$ & $3.5 \%$ \\
$H=0.6$ & 0.940 & 1.050 & 0.652 & $1.3 \%$ & $3.5 \%$ & 1.078 & 1.048 & 0.616 & $1.3 \%$ & $3.2 \%$ \\
$H=0.7$ & 1.077 & 1.035 & 0.586 & $1.6 \%$ & $3.9 \%$ & 1.189 & 1.031 & 0.556 & $1.4 \%$ & $3.1 \%$ \\
$H=0.8$ & 1.068 & 1.022 & 0.526 & $1.3 \%$ & $3.5 \%$ & 1.299 & 1.015 & 0.502 & $1.3 \%$ & $3.0 \%$
\end{tabular}

TABLE II

Same as in Table 1 but for MDMA.

\begin{tabular}{c|c|c|c|c|c|c|c|c|c|c}
\hline \hline$H \backslash C L$ & $D^{97.5 \%}$ & $\eta^{97.5 \%}$ & $\xi^{97.5 \%}$ & $\Delta_{M A E}^{97.5 \%}$ & $\Delta_{M E}^{97.5 \%}$ & $D^{95 \%}$ & $\eta^{95 \%}$ & $\xi^{95 \%}$ & $\Delta_{M A E}^{95 \%}$ & $\Delta_{M E}^{95 \%}$ \\
\hline$H=0.5$ & 1.924 & 1.052 & 0.866 & $1.7 \%$ & $4.6 \%$ & 2.656 & 1.053 & 0.869 & $1.4 \%$ & $4.0 \%$ \\
$H=0.6$ & 2.310 & 1.039 & 0.808 & $1.5 \%$ & $4.1 \%$ & 3.131 & 1.037 & 0.805 & $1.1 \%$ & $3.0 \%$ \\
$H=0.7$ & 2.719 & 1.026 & 0.751 & $1.4 \%$ & $3.4 \%$ & 3.675 & 1.024 & 0.749 & $1.3 \%$ & $3.6 \%$ \\
$H=0.8$ & 3.086 & 1.012 & 0.694 & $1.1 \%$ & $2.8 \%$ & 4.203 & 1.013 & 0.699 & $1.4 \%$ & $3.2 \%$
\end{tabular}

TABLE III

Results of the best fit for coefficients in Eq. (14) found for DFA-1 for series with various autocorrelation level measured by $H$ exponent and for chosen two confidence levels: $97.5 \%$ and $95 \%$.

\begin{tabular}{c|c|c|c|c|c|c|c|c|c|c}
\hline \hline$H \backslash C L$ & $A^{97.5 \%}$ & $A_{0}^{97.5 \%}$ & $B^{97.5 \%}$ & $\Delta_{M A E}^{97.5 \%}$ & $\Delta_{M E}^{97.5 \%}$ & $A^{95 \%}$ & $A_{0}^{95 \%}$ & $B^{95 \%}$ & $\Delta_{M A E}^{95 \%}$ & $\Delta_{M E}^{95 \%}$ \\
\hline$H=0.5$ & -6.02 & 6.023 & -92 & $1.8 \%$ & $5.1 \%$ & -7.00 & 7.003 & -100 & $1.8 \%$ & $5.1 \%$ \\
$H=0.6$ & -6.14 & 6.151 & -95 & $1.6 \%$ & $5.2 \%$ & -7.22 & 7.230 & -105 & $1.9 \%$ & $5.3 \%$ \\
$H=0.7$ & -6.46 & 6.472 & -97 & $1.9 \%$ & $4.6 \%$ & -7.66 & 7.668 & -103 & $1.8 \%$ & $4.6 \%$ \\
$H=0.8$ & -6.88 & 6.894 & -100 & $1.5 \%$ & $4.8 \%$ & -8.12 & 8.129 & -104 & $2.5 \%$ & $6.0 \%$
\end{tabular}

The answers can be deduced from plots shown in Figs. 9-10 (all plots made for variety of parameter values are qualitatively similar, so just exemplary dependencies are shown in here). Figure 9 shows the reproduced $H$ value as a function of a chosen scaling range for three discussed detrending methods and for two distinct lengths of random walk data. Figure 10 indicates the same dependence for a persistent signal. An answer to the second problem stated above concerning reproduction abilities of the input persistency level is given in the following Figs. 11-13.

One notices from Figs. 9-10 that DFA reproduces $H$ in the most stabile way but simultaneously underestimates its real value for persistent series. We have checked that for $H_{i n} \geq 0.7$ the outcome value of Hurst exponent determined within DFA will always lie below $H_{\text {in }}$ and this discrepancy grows with scaling range. The MDMA method reproduces input $H$ value less stabile than DFA but offers better performance in retrieving $H$ exponent than DMA. The MDMA reproduces $H$ exponent value better than DMA and worse than DFA for longer scaling ranges — independently on persistence level in data.
DMA and MDMA are not distinguishable for very short ranges $\left(\lambda \leq 10^{-1} L\right)$ but both methods slightly overestimate $H$ value for such scaling range. They underestimate $H$ for $\lambda>10^{-1} L$ but this underestimation is more gentle in case of MDMA, particularly for uncorrelated or weakly autocorrelated time series.

The most important results can be read from Fig. 11. These plots show the actual scaling range $\lambda^{*}$ for which the real value of $H$ (or $\gamma$ ) exponent is strictly reproduced. It turns out that for all detrending methods the power law relation seems to be valid

$$
\lambda^{*}=A L^{m},
$$

where the parameters $A$ and $m$ are found from the linear fit in double $\log$ scale and are collected in Table IV for four Hurst exponent values $H=0.5, H=0.6, H=$ $0.7, H=0.8$. The presented uncertainties in Table IV correspond to reproduction of $H$ at a very demanding level $|\delta H / H| \lesssim 1 \%$.

These results can be translated into a magnitude of relative percentage scaling range $\lambda^{*} / L$ shown further in Figs. 12-13, for which the input value of Hurst exponent is retrieved exactly. We may see that for short time 

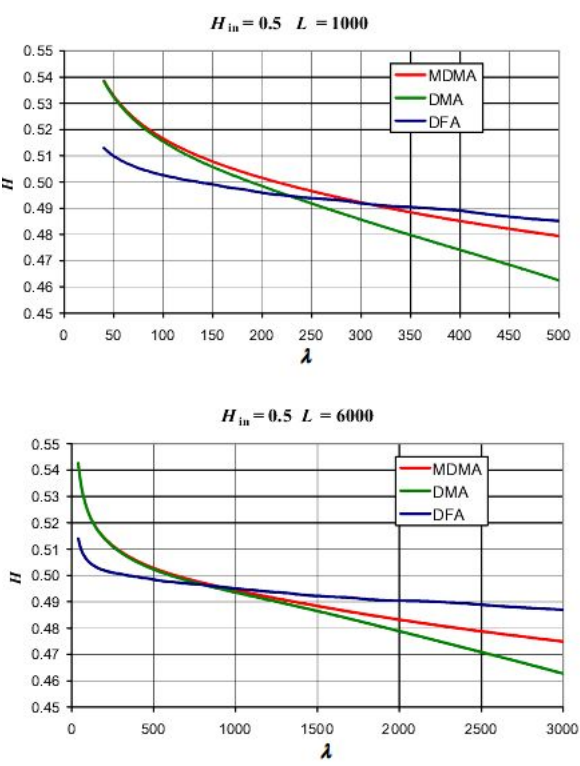

Fig. 9. Comparable reproduction of the Hurst exponent $H$ for integer Brownian motion within DFA, DMA and MDMA. The results for two length of data are shown $(L=1000$ and $L=6000)$.
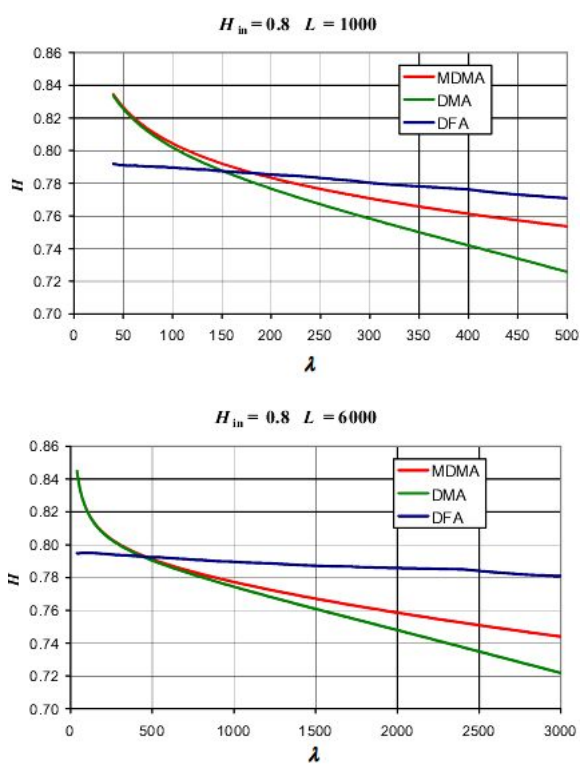

Fig. 10. Same as in Fig. 9 but for persistent synthetic signal with input scaling exponent value $H_{i n}=0.8$.

series $(L<2000)$ one needs to take longer scaling range $(10 \%-25 \% L)$ in case of DMA and MDMA to do so. The DFA is much less demanding here - if $L>2000$ it is sufficient to take even less than $10 \% L$ to calculate $H$ exactly. The data obtained from higher detrending polynomials (DFA-2, DFA-3) look quantitatively the same and have not been shown. It confirms the statement made in [24] that $H$ starts to depend more significantly on the polynomial order $p$ yet for $p>3$.

\section{Concluding Remarks}

Concluding, we may say that MDMA has always better scaling properties than DMA and usually better than
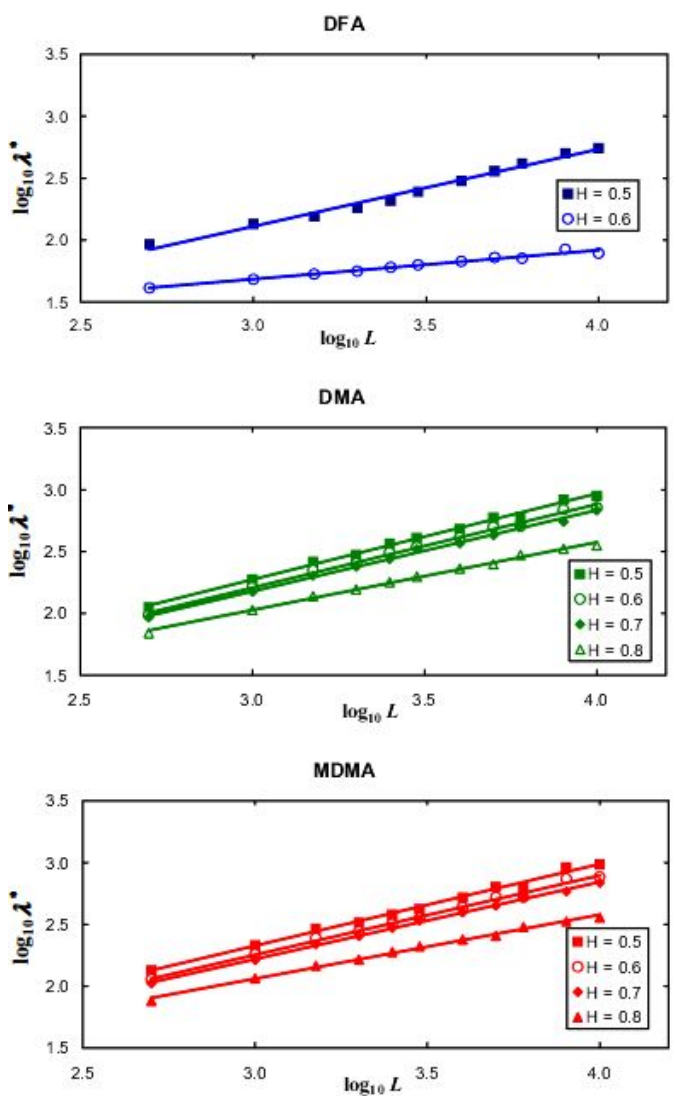

Fig. 11. Power law dependence between scaling range $\lambda$ well reproducing the true value of $H$ exponent for different kinds of fractional Brownian motion. DFA, DMA and MDMA results are compared together. The fitting parameters of power law dependence are collected with uncertainties in Table IV.
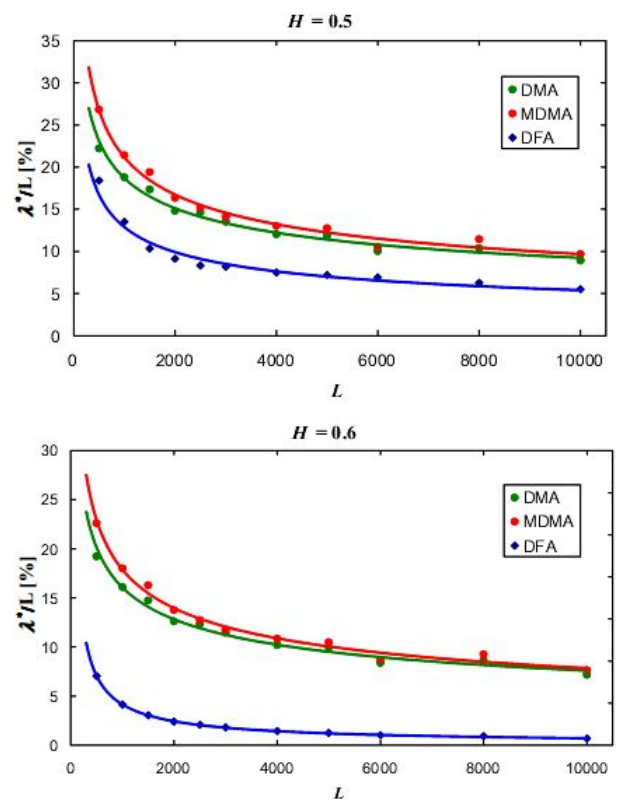

Fig. 12. Relative percentage scaling range $\lambda^{*} / L$ reproducing the input value of $H$ with uncertainty $|\delta H| / H \lesssim$ $1 \%$. The results for different detrending methods and for uncorrelated or moderately correlated data are shown. 

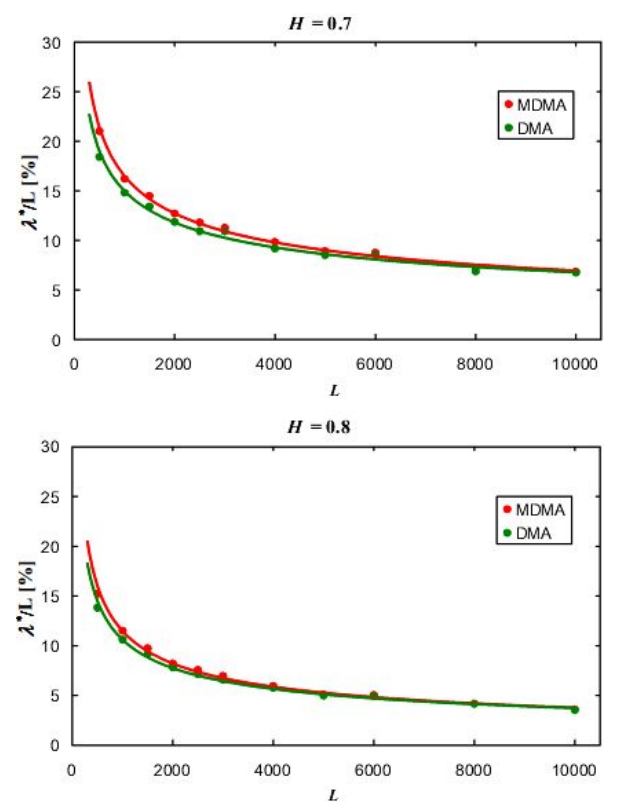

Fig. 13. Same as in Fig. 12 but for more persistant signals $(H=0.7$ and $H=0.8)$. Plots for DFA are absent since this method does not warranty solutions of the problem for such persistent data.

TABLE IV

Fit for parameters in Eq. (15) describing scaling range at which the actual input value of scaling exponent $H$ is well reproduced. The cross mark indicate that no solution is available for particular method. The uncertainties shown here come from the best fit estimation of $A$ and $m$ at $1 \sigma$ level in plots of Fig. 11.

\begin{tabular}{c|c|c|c}
\hline \hline Method & DFA & DMA & MDMA \\
\hline parameter & $\log \mathrm{A}$ & $\log \mathrm{A}$ & $\log \mathrm{A}$ \\
$\mathrm{H}=0.5$ & $0.240 \pm 0.082$ & $0.190 \pm 0.051$ & $0.343 \pm 0.061$ \\
$\mathrm{H}=0.6$ & $0.982 \pm 0.040$ & $0.180 \pm 0.055$ & $0.325 \pm 0.057$ \\
$\mathrm{H}=0.7$ & $\mathrm{X}$ & $0.207 \pm 0.046$ & $0.343 \pm 0.030$ \\
$\mathrm{H}=0.8$ & $\mathrm{X}$ & $0.381 \pm 0.042$ & $0.504 \pm 0.043$ \\
parameter & $\mathrm{m}$ & $\mathrm{m}$ & $\mathrm{m}$ \\
$\mathrm{H}=0.5$ & $0.623 \pm 0.023$ & $0.694 \pm 0.015$ & $0.661 \pm 0.018$ \\
$\mathrm{H}=0.6$ & $0.234 \pm 0.012$ & $0.675 \pm 0.016$ & $0.642 \pm 0.016$ \\
$\mathrm{H}=0.7$ & $\mathrm{X}$ & $0.656 \pm 0.013$ & $0.625 \pm 0.009$ \\
$\mathrm{H}=0.8$ & $\mathrm{X}$ & $0.548 \pm 0.012$ & $0.518 \pm 0.012$
\end{tabular}

DFA (for short $L$ and high requirements for $R^{2}$ ) what makes it superior in local analysis of series with changing (i.e., evolving) level of long-term memory. MDMA is overall somewhere between two other methods - it wins over DMA but loses with DFA. Only in the exceptional case of persistent data DFA is a loser, since MDMA and DMA are capable to reproduce correctly the input $H$ value for short scaling ranges $\left(\lambda \leq 10^{-1} L\right)$, while DFA method fails to do so.

Furthermore, DFA is most stabile but always underestimates the memory level $(H)$ for $H \geq 0.7$ and this discrepancy grows with $\lambda$. MDMA is less stabile than DFA but more stabile than DMA. MDMA reproduces $H$ exponent better than DMA and worse than DFA for longer scaling ranges independently on the memory level in signal. For short data length $(L<2000)$ DMA and MDMA allow to take longer scaling range (10\%-25\% of $L$ ) to exactly retrieve the input memory level. DFA does not offer this $(\simeq 1 \%-5 \%$ of $L$ for $H<0.7$ and fails for $H \geq 0.7)$.

Finally, a winner is not easy defined. MDMA and DMA are with no doubts number one for small $L$ and high $R^{2}$, DFA is number one for longer series with a smaller memory level $(0.5<H<0.7)$, while an advantage of MDMA is seen for longer signals with substantial memory level.

\section{References}

[1] Y.-H. Shao, G.-F. Gu, Z.-Q. Jiang, W.-X. Zhou, D. Sornette, Scientific Reports 2, 835 (2012).

[2] R.M. Bryce, K.B. Sprague, Scientific Reports 2, 315 (2012).

[3] L. Xu, P.Ch. Ivanov, K. Hu, Z. Chen, A. Carbone, H.E. Stanley, Phys. Rev. E 71, 051101 (2005).

[4] S. Michalski, Physica A 387, 217 (2008).

[5] A. Bashan, R. Bartsch, J.W. Kantelhardt, S. Havlin, Physica A 387, 5080 (2008).

[6] R. Weron, Physica A 312, 285 (2002).

[7] M. Couillard, M. Davison, Physica A 348, 404 (2005).

[8] M.S. Taqqu, V. Teverovsky, W. Willinger, Fractals 3, 785, (1995).

[9] B.B. Mandelbrot The Fractal Geometry of Nature, W.H. Freeman, New York 1982.

[10] H.E. Hurst, Trans. Am. Soc. Civ. Eng. 116, 770 (1951).

[11] B.B. Mandelbrot, J.R. Wallis, Water Resour. Res. 5 321 (1969).

[12] J.W. Kantelhardt, E. Koscielny-Bunde, H.H.A. Rego, S. Havlin, A. Bunde, Physica A 295, 441 (2001).

[13] C.-K. Peng, S. Havlin, H.E. Stanley, A.L. Goldberger, Chaos 5, 82 (1995).

[14] C.-K. Peng, S.V. Buldyrev, S. Havlin, M. Simons, H.E. Stanley, and A.L. Goldberger, Phys. Rev. E 49, 1685 (1994).

[15] E. Alessio, A. Carbone, G. Castelli, V. Frappietro, Eur. Phys. J. B 27, 197 (2002).

[16] A. Carbone, G. Castelli, H.E. Stanley, Phys. Rev. E 69, 026105 (2004).

[17] A. Carbone, H.E. Stanley, Physica A 340, 544 (2004).

[18] A. Carbone, G. Castelli, H.E. Stanley, Physica A 344, 267 (2004).

[19] J.W. Kantelhardt, S.A. Zschiegner, E. KoscielnyBunde, S. Havlin, A. Bunde, H.E. Stanley, Physica A 316, 87 (2002).

[20] D. Grech, Z. Mazur, Phys. Rev. E 87, 052809 (2013).

[21] H.A. Makse, S. Havlin, M. Schwartz, H.E. Stanley, Phys. Rev. E 53, 5445 (1996).

[22] D. Grech, G. Pamuła, Physica A 392, 5845 (2013).

[23] D. Grech, Z. Mazur, Physica A 392, 2384 (2013).

[24] P. Oświęcimka, S. Drożdż, J. Kwapień, A.Z. Górski, Acta Phys. Pol. A 123, 597 (2013). 